\title{
ON POINTWISE APPROXIMATION OF FOURIER SERIES BY TYPICAL MEANS
}

\author{
HUBERT BERENS
}

(Received February 2, 1970)

1. Notations and results. Throughout the paper $f(x)$ will be a real-valued, $2 \pi$-periodic, $L$-integrable function on the real line such that $\int_{-\pi}^{\pi} f(x) d x=0$. We denote the Fourier series of $f$ by

$$
S(f) \sim \sum_{k=1}^{\infty} A_{k}(f ; x), \quad A_{k}(f ; x)=a_{k} \cos k x+b_{k} \sin k x
$$

where $a_{k}$ and $b_{k}$ are its Fourier coefficients.

Let $X_{2 \pi}$ be one of the function spaces $C_{2 \pi}$, or $L_{2 \pi}^{p}, 1 \leqq p<\infty$, of the functions in question; the spaces are endowed with their usual norms. Let $\gamma$ be a positive constant. If for an $f$ in $X_{2 \pi}$ the associated series

$$
\sum_{k=1}^{\infty} k^{\gamma} A_{k}(f ; x)
$$

is the Fourier series of some function $g$ in $X_{2 \pi}$, we say that $f$ has a Riesz derivative of order $\gamma$ in $X_{2 \pi}$ and we write

$$
D^{[\gamma]} f:=f^{[\gamma]}=g
$$

It is well known (see e.g., P. L. Butzer-K. Scherer [3, Ch. 4]) that the operator $D^{[\gamma]}$ is closed with domain

$$
X_{2 \pi}^{[\gamma]}=\left\{f \in X_{2 \pi}: \sum_{k=1}^{\infty} k^{\gamma} A_{k}(f ; x) \text { is the Fourier series of a function } g \text { in } X_{2 \pi}\right\}
$$

dense in $X_{2 \pi}$. Since for an $f \in X_{2 \pi}^{[\gamma]}, D^{[\gamma]} f=0$ implies $f=0, D^{[\gamma]}$ has an inverse $I^{\text {[r] }}$. Its extension to the whole space $X_{2 \pi}$ is the so-called Riesz potential of order $\gamma$. With $I^{\{\gamma\}} f:=f_{[\gamma\}}$ (or: $=f^{\{-\gamma\}}$ )we have

$$
S\left(f_{[\gamma\}}\right) \sim \sum_{k=1}^{\infty} \frac{A_{k}(f ; x)}{k^{\gamma}} .
$$


The typical means of order $\gamma$

$$
P_{n, \gamma}(f ; x)=\sum_{k=1}^{n}\left(1-\frac{k^{\gamma}}{(n+1)^{\gamma}}\right) A_{k}(f ; x) \quad(\gamma>0, n=1,2, \cdots)
$$

of the Fourier sereis of a function $f$ are closely related to its Riesz derivative $f^{[\gamma]}$. Indeed, the following theorem holds true.

THEOREM A. Let $\gamma>0$. If for the functions $f$ and $g$ in $X_{2 \pi}$

$$
\lim _{n \rightarrow \infty}\left\|(n+1)^{\gamma}\left\{R_{n, \gamma} f-f\right\}-g\right\|_{X_{2 \pi}}=0,
$$

then $f \in X_{2 \pi}^{[\gamma]}$ and $f^{[\gamma]}=-g$, and vice versa. If in particular $g=0$, then $f$ is the zero-function.

The theorem is a modification of the saturation theorem for the typical means in $X_{2 \pi}$. It has its roots in results due to A. Zygmund and B. Sz.-Nagy in the 1940's. The theorem itself was first formulated and proved by S. Aljanciic for the space $C_{2 \pi}$ and by G. Sunouchi-C. Watari for the spaces in question in the late 1950's. In the form of Theorem A it is due to P. L. Butzer-E. Görlich. For details we refer to P. L. Butzer-K. Scherer, loc. cit. Finally, we have to mention that G. Sunouchi [4] studied local versions of Theorem A.

It is the aim of this note to prove the following pointwise analogue of Theorem A.

THEOREM B. Let $f \in L_{2 \pi}$ be such that

$$
\lim _{n \rightarrow \infty} R_{n, \gamma}(f ; x)=f(x)
$$

finitely for all $x$ in some interval $(a, b)$. If there exists a finitely-valued, L-integrable function $g(x)$ in $(a, b)$ such that

$$
\lim _{n \rightarrow \infty}(n+1)^{\gamma}\left\{R_{n, \gamma}(f ; x)-f(x)\right\}=g(x)
$$

pointwise for all $x$ in $(a, b)$, then $f^{(r-2)}$ belongs to $L_{2 \pi}$ and for almost all $x$ in $(a, b)$

$$
f^{(\gamma-2\}}(x)=A x+B+\int_{a}^{x} d t \int_{a}^{t} g(u) d u
$$


where $A$ and $B$ are some constants.

For $0<\gamma<1$, ( 3 ) remains true even if (1) is violated in a denumerable set $E$ of points, supposed that

$$
\left|R_{n, \gamma}(f ; x)\right|=o\left(n^{1-\gamma}\right) \quad(\text { for all } x \in E) .
$$

For $\gamma=1,(3)$ remains true even if (2) is violated in a denumerable set $E$ of points.

For $\gamma>1,(3)$ remains true even if (2) is violated in a denumerable set of points E, supposed that

$$
\left|R_{n, \gamma}(f ; x)-f(x)\right|=o\left(n^{1-\gamma}\right) \quad(\text { for all } x \in E) .
$$

For $\gamma=1$ (Fejér means) and $g(x)=0$, Theorem B is due to V. A. Andrienko [1].

The following corollaries are obvious consequences of Theorem B.

COROLLARY 1. Let $f$ and $g$ be finitely-valued functions in $L_{2 \pi}$ such that the conditions (1) and (2) of Theorem $B$ are satisfied for all $x$ except in a denumerable set $E$ of points at which (4) holds, then $f \in L_{2 \pi}^{\{\gamma\}}$ and for almost all $x, f^{[\gamma]}(x)=-g(x)$.

COROLLARY 2. Let $f$ and $g$ belong to $C_{2 \pi}$ such that

$$
\lim _{n \rightarrow \infty}(n+1)^{\gamma}\left\{R_{n, \gamma}(f ; x)-f(x)\right\}=g(x)
$$

pointwise everywhere, then $f \in C_{2 \pi}^{\{\gamma]}$ and $f^{[\gamma]}=-g$, and vice versa.

The second corollary substantially weakens the statement of Theorem A for the space $X_{2 \pi}=C_{2 \pi}$.

REMARK. The function $f_{\gamma}$ given by

$$
S\left(f_{\gamma}\right) \sim \sum_{k=1}^{\infty} \frac{\cos k x}{k^{\gamma}} \quad(\gamma>0)
$$

shows that if there is only one exceptional point $x_{0}(\bmod 2 \pi)$ in $R$ for which (4) is violated then the statement of Corollary 1 is wrong. Indeed, the series (5) converges for all $x \neq 0(\bmod 2 \pi)$ and the associated function $f_{\gamma}$ belongs to 
$L_{2 \pi}$. Moreoevr, for all $x \neq 0(\bmod 2 \pi)$

$$
\lim _{n \rightarrow \infty}(n+1)^{\gamma}\left\{R_{n, \gamma}\left(f_{\gamma} ; x\right)-f_{\gamma}(x)\right\}=\frac{1}{2},
$$

while for $x_{0}=0(\bmod 2 \pi)$

$$
\left|R_{n, \gamma}\left(f_{\gamma} ; x_{0}\right)\right|= \begin{cases}\Omega\left(n^{1-\gamma}\right)(n \rightarrow \infty), & 0<\gamma<1, \\ \Omega(\log n)(n \rightarrow \infty), & \gamma=1,\end{cases}
$$

and

$$
\left|R_{n, \gamma}\left(f_{\gamma} ; x_{0}\right)-f\left(x_{0}\right)\right|=\Omega\left(n^{1-\gamma}\right)(n \rightarrow \infty), \gamma>1
$$

We conclude this section with the formulation of a third theorem although it seems to be known, see G. Sunouchi [4]. The proof follows directly from relation (11) and the Fejér-Lebesgue theorem.

THEOREM C. Let $f$ be a function in $L_{2 \pi}^{[\gamma]}$. For almost all $x$

$$
\lim _{n \rightarrow \infty}(n+1)^{\gamma}\left\{R_{n, \gamma}(f ; x)-f(x)\right\}=-f^{[\gamma]}(x) .
$$

2. Proof of Theorem B. The proof is based on two lemmas. Lemma 1 is a uniqueness theorem for $(\mathrm{C}, 1)$-summable trigonometric series. It can be obtained out of Verblunsky's uniqueness thoerems for Abel summable trigonometric series (cf. A. Zygmund [6, p. 352ff]). Moreover, Lemma 1 is a very special form of results due to $\mathrm{F}$. Wolf [5] about $(C, \lambda)$-summable series. In Lemma 2 we rewrite condition (2) so that Theorem B can be concluded directly out of Lemma 1.

LEMMA 1. Let $\sum_{k=1}^{\infty} A_{k}(x)$ be a trigonometric series. If in some interval $(a, b)$ the limit

$$
\lim _{n \rightarrow \infty} \sigma_{n}(x):=\lim _{n \rightarrow \infty} \sum_{k=1}^{n}\left(1-\frac{k}{n+1}\right) A_{k}(x)=g(x)
$$

exists finitely, except in a denumerable set $E$, with $g(x)$ L-integrable, and if for all $x \in E$

$$
\sigma_{n}(x)=o(n) \quad(n \rightarrow \infty)
$$


then $\sum_{1}^{\infty} A_{k}(x) / k^{2}$ is the Fourier series of some function $F$ in $L_{2 \pi}$ and for almost all $x$ in $(a, b)$

$$
F(x)=A x+B+\int_{a}^{x} d t \int_{a}^{t} g(u) d u .
$$

If, moreover, the interval $(a, b)$ contains $[0,2 \pi)$ then $\sum_{1}^{\infty} A_{k}(x)$ is the Fourier series of $g$.

LEMMA 2. Let $f$ in $L_{2 \pi}$ be such that for some $x, R_{n, \gamma}(f ; x) \rightarrow c(x)$ finitely as $n \rightarrow \infty$. The limit

$$
\lim _{n \rightarrow \infty}(n+1)^{\gamma}\left\{R_{n, \gamma}(f ; x)-c(x)\right\}
$$

exists finitely if, and only if, the limit

$$
\lim _{n \rightarrow \infty}-\sigma_{n}^{\{\gamma\}}(f ; x):=\lim _{n \rightarrow \infty} \sum_{k=1}^{n}\left(1-\frac{k}{n+1}\right)\left(-k^{\gamma}\right) A_{k}(f ; x)
$$

exists, and both limits are equal.

PROOF. By the use of the identity

$$
R_{n-1, \gamma}(f ; x)-R_{n, \gamma}(f ; x)=\left\{\frac{1}{n^{\gamma}}-\frac{1}{(n+1)^{\gamma}}\right\} \sum_{k=1}^{n}\left(-k^{\gamma}\right) A_{k}(f ; x)(n=1,2, \cdots),
$$

see P. L. Butzer-S. Pawelke [2], we obtain the relation

$$
R_{n, \gamma}(f ; x)-c(x)=\sum_{k=n+1}^{\infty}\left\{\frac{1}{k^{\gamma}}-\frac{1}{(k+1)^{\gamma}}\right\} \sum_{j=1}^{k}\left(-j^{\gamma}\right) A_{j}(f ; x) .
$$

Introducing the abbreviations $s_{n}=\sum_{k=1}^{n}\left(-k^{\gamma}\right) A_{k}(f ; x)$ and $t_{n}=R_{n, \gamma}(f ; x)-c(x)$, the latter equation simply reads

$$
t_{n}=\sum_{k=n+1}^{\infty}\left\{\frac{1}{k^{\gamma}}-\frac{1}{(k+1)^{\gamma}}\right\} s_{k} .
$$


It is appropriate to use two additional notations : $\tau_{n}=(n+1)^{\gamma} t_{n}$ and $\sigma_{n}=\left(\sum_{k=1}^{n} s_{k}\right)$ $/(n+1)$.

To prove the "if"-part, we have to show that $\sigma_{n} \rightarrow s$ as $n \rightarrow \infty$ implies $\tau_{n} \rightarrow s$ as $n \rightarrow \infty$. Indeed, by partial summation of the sum on the right-hand side of (10) we have

$$
\begin{aligned}
\tau_{n}= & (n+1)^{\gamma} \sum_{k=n+1}^{\infty}(k+1)\left\{\frac{1}{k^{\gamma}}-\frac{2}{(k+1)^{\gamma}}+\frac{1}{(k+2)^{\gamma}}\right\} \sigma_{k} \\
& -(n+1)^{1+\gamma}\left\{\frac{1}{(n+1)^{\gamma}}-\frac{1}{(n+2)^{\gamma}}\right\} \sigma_{n},
\end{aligned}
$$

and the result follows by taking into account that

$$
\begin{aligned}
1= & (n+1)^{\gamma} \sum_{k=n+1}^{\infty}(k+1)\left\{\frac{1}{k^{\gamma}}-\frac{2}{(k+1)^{\gamma}}+\frac{1}{(k+2)^{\gamma}}\right\} \\
& -(n+1)^{1+\gamma}\left\{\frac{1}{(n+1)^{\gamma}}-\frac{1}{(n+2)^{\gamma}}\right\}
\end{aligned}
$$

identically in $n=0,1,2, \cdots$ and that

$$
\lim _{n \rightarrow \infty}(n+1)^{1+\gamma}\left\{\frac{1}{(n+1)^{\gamma}}-\frac{1}{(n+2)^{\gamma}}\right\}=\gamma .
$$

On the other hand, by (10)

$$
t_{n-1}-t_{n}=\left\{\frac{1}{n^{\gamma}}-\frac{1}{(n+1)^{\gamma}}\right\} s_{n}:=\frac{1}{C_{n}} s_{n} \quad(n=1,2, \cdots)
$$

Setting $s_{0}$ as well as the constant $C_{0}$ equal to zero, we obtain again by partial summation

$$
\begin{aligned}
\sum_{k=0}^{n} s_{k} & =s_{0}+\sum_{k=1}^{n} C_{k}\left(t_{k-1}-t_{k}\right) \\
& =s_{0}+\sum_{k=0}^{n}\left(C_{k+1}-C_{k}\right) t_{k}-C_{n+1} t_{n}
\end{aligned}
$$

or 


$$
\sigma_{n}=\frac{1}{n+1} \sum_{k=0}^{n} \frac{C_{k+1}-C_{k}}{(k+1)^{\gamma}} \tau_{k}-\frac{C_{n+1}}{(n+1)^{1+\gamma}} \tau_{n} .
$$

Since

$$
1=\frac{1}{n+1}+\frac{1}{n+1} \sum_{k=0}^{n} \frac{C_{k+1}-C_{k}}{(k+1)^{\gamma}}-\frac{C_{n+1}}{(n+1)^{1+\gamma}}
$$

identically in $n=0,1,2, \cdots$, and since $\lim _{n \rightarrow \infty} C_{n+1} /(n+1)^{1+\gamma}=\frac{1}{\gamma}$ (see above), it is easy to conclude that $\tau_{n} \rightarrow s$ as $n \rightarrow \infty$ implies $\sigma_{n} \rightarrow s$ as $n \rightarrow \infty$. This proves the “only if"-part.

The proof of Theorem $\mathrm{B}$ now follows by setting the coefficients $A_{k}(x)$ in Lemma 1 equal to $\left(-k^{\gamma}\right) A_{k}(f ; x), k=1,2, \cdots$, i. e., $\sigma_{n}(x)=-\sigma_{n}^{[\gamma]}(f ; x)$.

With respect to the conditions upon $R_{n, \gamma}(f ; x)$ at the points $x$ in the exceptional set $E$, we have to mention that for $0<\gamma<1$ (4a) is equivalent to ( 7 ), for $\gamma=1$ (1) implies ( 7 ), and for $\gamma>1$ again (4b) is equivalent ( 7 ).

\section{REFERENCES}

[1] A. V. ANDRIENKo, Approximation of functions by Fejér means, Siberian Math. J., 9 (1968), 1-8.

[2] P. L. BUTZER AND S. PAWELKE, Ableitungen von trigonometrischen Approximationsprozessen, Acta Sci. Math. (Szeged), 28(1967), 173-183.

[3] P. L. BUTZER AND K. SCHERER, Approximationsprozesse und Interpolationsmethoden. B.IHochschulskripten Bd. 826/826a. Mannheim, 1968.

[4] G. SUNOUCHI, On the class of saturation in the theory of approximation II, III. Tôhoku Math. J., 13(1961), 112-118; 320-328.

[5] F. WOLF, On summable trigonometric series: An extenison of uniqueness theorems, Proc. London Math. Soc. (2), 45(1939), 328-356.

[6] A. ZYGMUND, Trigonometric Series. Vol. I. Camibrdge, 1959.

DEPARTMENT OF MATHEMATICS

UNIVERSITY OF CALIFORNIA

SANTA BARBARA, CALIFORNIA 
